\title{
Facteurs Épidémiologiques et Pronostiques Associés Aux Étiologies Des Hémorragies Digestives Hautes au CHU Campus de Lomé
}

\author{
Aklesso Bagny \\ Service d'Hépato-Gastroentérologie, CHU Campus, Lomé, Togo \\ Département d'Hépato-Gastroentérologie, Université de Lomé, Togo \\ Lidawu Roland-Moise Kogoe \\ Laconi Yeba Kaaga \\ Service d'Hépato-Gastroentérologie, CHU Campus, Lomé, Togo \\ Late Mawuli Lawson-Ananissoh \\ Service d'Hépato-Gastroentérologie, CHU Campus, Lomé, Togo \\ Département d'Hépato-Gastroentérologie, Université de Lomé, Togo \\ Debehoma Redah \\ Mawunyo Henoc Gbolou \\ Yendoukoa Yves Kanake \\ Service d'Hépato-Gastroentérologie, CHU Campus, Lomé, Togo
}

Doi:10.19044/esj.2021.v17n34p44

Submitted: 18 August 2021

Accepted: 07 September 2021

Published: 30 September 2021
Copyright 2021 Author(s)

Under Creative Commons BY-NC-ND

4.0 OPEN ACCESS

Cite As:

Bagny A., Roland-Moise Kogoe L., Yeba Kaaga L., Lawson-Ananissoh L.M., Redah D., Gbolou H.M. \& Kanake Y.Y. (2021). Facteurs Épidémiologiques et Pronostiques Associés Aux Étiologies Des Hémorragies Digestives Hautes Au CHU Campus de Lomé. European Scientific Journal, ESJ, 17(34), 44.

https://doi.org/10.19044/esj.2021.v17n34p44

\section{Résumé}

Objectif : Décrire les aspects épidémiologique, clinique et pronostique associés aux étiologies des hémorragies digestives hautes au CHU Campus de Lomé

Patients et méthode: Etude transversale à collecte rétrospective, à visée descriptive et analytique menée du 1er Janvier 2014 au 31 Décembre 2019. Le seuil de significativité était retenu pour $\mathrm{p}<0,05$.

Résultats: Deux cent cinquante et un patients avaient été inclus. L'hémorragie était d'origine hypertensive portale chez 69 patients (27,71\%) ; ulcéreuse gastro-duodénale chez 100 patients (39,84\%). Chez 25 patients 
(9,96\%), la fibroscopie œsogastroduodénale était normale. Une rupture de varices œsophagiennes était retrouvée chez 98,55\% des patients présentant une hypertension portale. Les ulcères gastroduodénaux représentaient 54,94\% des hémorragies digestives hautes d'origine non hypertensive portale. La valeur moyenne du score de Rockall était de $4( \pm 1)$ chez les patients présentant une hémorragie d'origine hypertensive et de $3( \pm 1)$ chez les patients avec hémorragie non hypertensive portale $(\mathrm{p}<0,001)$. La valeur moyenne du score de Glasgow-Blatchford était de $10( \pm 3)$ chez les patients présentant une hémorragie d'origine hypertensive et $9( \pm 3)$ chez les patients avec hémorragie digestive haute d'origine non hypertensive $(\mathrm{p}<0,001)$. La récidive hémorragique et le décès étaient survenus chez les patients présentant un saignement d'origine hypertensive portale dans respectivement 54,84\% $(\mathrm{p}<0,001)$ et $71,42 \%(\mathrm{p}<0,001)$.

Conclusion: Les lésions inflammatoires aiguës et chroniques représentent la première étiologie des hémorragies digestives hautes dans le service d'Hépato-gastroentérologie du CHU Campus. Ces hémorragies sont associées à la prise de médicaments gastrotoxiques et à un moindre risque de récidive hémorragique et de décès.

Mots clés : Hémorragie Digestive Haute, Etiologie, Pronostique, Lomé, Togo

\title{
Epidemiological and Prognostic Factors Associates with Upper Gastrointestinal Bleeding in Campus Teaching Hospital of Lome
}

\author{
Aklesso Bagny \\ Service d'Hépato-Gastroentérologie, CHU Campus, Lomé, Togo \\ Département d'Hépato-Gastroentérologie, Université de Lomé, Togo \\ Lidawu Roland-Moise Kogoe \\ Laconi Yeba Kaaga \\ Service d'Hépato-Gastroentérologie, CHU Campus, Lomé, Togo \\ Late Mawuli Lawson-Ananissoh \\ Service d'Hépato-Gastroentérologie, CHU Campus, Lomé, Togo \\ Département d'Hépato-Gastroentérologie, Université de Lomé, Togo \\ Debehoma Redah \\ Mawunyo Henoc Gbolou \\ Yendoukoa Yves Kanake \\ Service d'Hépato-Gastroentérologie, CHU Campus, Lomé, Togo
}




\section{Abstract}

Objective: To describe epidemiological et prognostic outcomes associated with etiologies upper gastrointestinal bleeding in Campus Teaching Hospital of Lome

Patients and method: Cross-sectional study with retrospective collection, descriptive and analytical aim carried out from January 1, 2014 to December 31, 2019.

Results: Two hundred and one patients were included. The hemorrhage was of portal hypertensive origin in 69 patients (27.71\%); peptic ulcer in 100 patients (39.84\%). In 25 patients (9.96\%), the oesogastroduodenal fibroscopy was normal. Ruptured esophageal varices were found in $98.55 \%$ of patients with portal hypertension. Peptic ulcers accounted for $54.94 \%$ of upper GI bleeding of non-portal hypertensive origin. The mean value of the Rockall score was $4( \pm 1)$ in patients with hemorrhage of hypertensive origin and $3( \pm 1)$ in patients with non-portal hypertensive hemorrhage $(\mathrm{p}<0.001)$. The mean Glasgow-Blatchford score was $10( \pm 3)$ in patients with hemorrhage of hypertensive origin and $9( \pm 3)$ in patients with upper GI hemorrhage of nonhypertensive origin $(\mathrm{p}<0.001)$. Hemorrhagic recurrence and death occurred in patients with bleeding of hypertensive origin in $54.84 \%(\mathrm{p}<0.001)$ and $71.42 \%(\mathrm{p}<0.001)$ respectively.

Conclusion: Acute and chronic inflammatory lesions represent the first etiology of upper GI bleeding in the Gastroenterology Department of the Campus Teaching Hospital of Lome. These hemorrhages are associated with the use of gastrotoxic drugs and with a lower risk of recurrence of hemorrhage and death.

Keywords: Upper Gastrointestinal Bleeding, Etiology, Prognosis, Lome, Togo

\section{Introduction}

L'hémorragie digestive haute (HDH) est un saignement dont l'origine est localisée sur la portion du tube digestif située en amont de l'angle de Treitz. Cette hémorragie peut s'extérioriser par une hématémèse ou un méléna (Lasserre $\mathrm{N}$ et al., 2009). C’est une urgence médico-chirurgicale nécessitant une prise en charge minutieuse. L'incidence annuelle est estimée à 100-150 épisodes pour 100000 habitants (Hervé S, 2007; Lasserre N et al., 2009). Les étiologies des $\mathrm{HDH}$ sont classées en deux catégories: les hémorragies digestives hautes d'origine hypertensive, et les hémorragies digestives hautes d'origine non-hypertensives (Czernichow $\mathrm{P}$ et al., 2000). Les HDH d'origine hypertensive portale sont une complication majeure de la cirrhose (D'amigo \& De Franchis, 2003). En 2012, une étude menée (Bagny et al., 2012) avait permis de déterminer le profil étiologique des hémorragies digestives hautes, 
sans toutefois décrire les éléments épidémiologique et pronostique propres aux étiologies retrouvées. Ainsi, avait-il semblé nécessaire de mener notre étude avec comme objectif de décrire les aspects épidémiologique et pronostique associés aux étiologies des hémorragies digestives hautes au CHU Campus de Lomé.

\section{Patients et méthode}

Il s'agit d'une étude transversale à collecte rétrospective, à visée descriptive et analytique menée du $1^{\text {er }}$ Janvier 2014 au 31 Décembre 2019. Avaient été inclus tous les patients hospitalisés dans le service d’Hépatogastroentérologie du CHU Campus pour hémorragie digestive haute, ou ayant présenté une hémorragie digestive haute en cours d'hospitalisation, pendant la période d'étude; et ayant réalisé une fibroscopie œsogastroduodénale. L'hémorragie digestive haute était définie par la présence d'une hématémèse et/ou d'un méléna. Les patients présentant une instabilité hémodynamique avaient initialement bénéficié de mesures de réanimation médicale avant réalisation de la fibroscopie œsogastroduodénale. N'avaient été admis en salle de fibroscopie que les patients stables sur le plan hémodynamique.

Les caractéristiques épidémiologiques, cliniques, évolutives et les résultats endoscopiques ont été recueillies à l'aide d'une fiche conçue sous Microsoft Word version 2010 et entrées dans un masque Epidata 3.1. Les scores de Rockall et de Glasgow-Blatchford avaient permis d'évaluer le pronostic des patients. Ces données ont été traitées et apurées à l'aide du logiciel Stata version 2013.

L'analyse comprenait un volet descriptif de l'ensemble des patients puis des deux groupes étiologiques; puis un volet analytique. L'étude analytique avait consisté à rechercher l'existence de facteurs associés aux différents groupes étiologiques. Les données quantitatives ont été présentées sous forme de moyenne, d'écart-type et d'intervalles. Les données qualitatives ont été présentées sous formes d'effectifs et de pourcentages. Les tests t de Student, exact de Fischer et Chi-carré ont été appliqués aux différents paramètres. Le seuil de significativité était retenu pour $\mathrm{p}<0,05$.

\section{Résultats}

Au total 4772 patients avaient été admis dans le service durant la période d'étude. Parmi ces patients, 314 présentaient une hémorragie digestive haute (fréquence hospitalière $=6,58 \%$ ) ; et 251 remplissaient les critères d'inclusion.

\section{Caractéristiques de la population d'étude}

L'âge moyen de la population d'étude était de $45,34( \pm 16,72)$ ans [15ans - 95 ans]. L'étude retrouvait 182 sujets de sexe masculin (72,80\% ; 
sex-ratio $\mathrm{H} / \mathrm{F}=2,64)$. L'âge moyen chez les hommes était de 43,36( $\pm 15,61)$ ans, contre $50,61( \pm 18,49)$ chez les femmes (IC à $95 \%=[43,25-47,42]$; $\mathrm{p}=0,002$ ). La tranche d'âge des [30 ans - 40 ans[ était la plus représentée. (Tableau 1).

Tableau 1. Caractéristiques Épidémiologiques, Cliniques Et Évolutifs

\begin{tabular}{|c|c|c|c|c|c|c|c|c|c|c|c|}
\hline & & \multicolumn{3}{|c|}{ Population d'étude } & \multicolumn{3}{|c|}{$\begin{array}{l}\text { Hémorragie d'origine } \\
\text { hypertensive portale }\end{array}$} & \multicolumn{3}{|c|}{$\begin{array}{c}\text { Hémorragie d'origine } \\
\text { non hypertensive } \\
\text { portale }\end{array}$} & \multirow[t]{2}{*}{ p } \\
\hline & & $\mathbf{n}$ & $\%$ & Moyenne & $\mathbf{n}$ & $\%$ & Moyenne & $\mathbf{n}$ & $\%$ & $\begin{array}{c}\text { Moyenn } \\
\text { e }\end{array}$ & \\
\hline \multirow[t]{2}{*}{ Sexe } & Masculin & 182 & 72,80 & - & 51 & 75 & - & 130 & 72,22 & - & \multirow[t]{2}{*}{0,339} \\
\hline & Féminin & 69 & 27,20 & - & 17 & 25 & - & 51 & 27,78 & - & \\
\hline Age & & & & $\begin{array}{c}45,34 \pm 16,7 \\
2 \\
\end{array}$ & & & $\begin{array}{c}43,11 \pm 13 \\
96\end{array}$ & & & $\begin{array}{c}46,05 \pm 17 \\
, 64\end{array}$ & 0,219 \\
\hline \multicolumn{2}{|c|}{$\begin{array}{l}\text { Hépatopathie } \\
\text { chronique }\end{array}$} & 29 & 11,65 & & 27 & 39,71 & & 2 & 1,12 & & $<0,001$ \\
\hline \multicolumn{2}{|c|}{ Ethylisme } & 85 & 34,14 & & 26 & 38,24 & & 59 & 32,96 & & 0,436 \\
\hline \multicolumn{2}{|c|}{ Tabagisme } & 11 & 5,58 & & 2 & 3,29 & & 9 & 6,52 & & 0,381 \\
\hline \multicolumn{2}{|c|}{ Prise d'AINS } & 82 & 32,93 & & 11 & 16,18 & & 70 & 39,11 & & 0,001 \\
\hline \multicolumn{2}{|c|}{$\begin{array}{c}\text { Ulcère } \\
\text { gastroduodénal }\end{array}$} & 18 & 7,73 & & 2 & 3,03 & & 16 & 9,64 & & 0,090 \\
\hline \multicolumn{2}{|c|}{ Glasgow-Blatchford } & & & $9 \pm 3$ & & & $10 \pm 3$ & & & $8 \pm 3$ & $<0,001$ \\
\hline \multicolumn{2}{|c|}{ Rockall } & & & $4 \pm 1$ & & & $4 \pm 1$ & & & $3 \pm 1$ & $<0,001$ \\
\hline \multicolumn{2}{|c|}{$\begin{array}{c}\text { Récidive } \\
\text { hémorragique }\end{array}$} & 31 & 12,35 & & 17 & 24,64 & & 14 & 7,78 & & $<0,001$ \\
\hline \multicolumn{2}{|c|}{ Décès } & 14 & 5,58 & & 10 & 14,49 & & 4 & 2,22 & & $<0,001$ \\
\hline
\end{tabular}

L'hémorragie digestive était extériorisée à type d'hématémèse associée à un méléna dans $87,10 \%$ des cas, de méléna isolé dans 12,90\% des cas. Les antécédents retrouvés étaient: l'existence d'une hépatopathie chronique (11,65\%), une notion de prise d'anti-inflammatoire avait été retrouvée dans 55,38\% des cas. A l'admission, 70,93\% présentaient un retentissement hémodynamique ayant nécessité la mise en route de mesures de réanimation spécifiques.

\section{Etiologies des hémorragies digestives hautes}

L'exploration endoscopique avait permis de retrouver comme origine du saignement : une hypertension portale chez 69 patients $(27,71 \%)$; une maladie ulcéreuse gastro-duodénale chez 100 patients (39,84\%), des lésions aiguës de la muqueuse gastroduodénale chez 67 patients $(26,69 \%)$; un Mallory-Weiss chez 11 patients (4,38\%), une œsophagite chez 9 patients (3,59\%), un cancer gastrique chez 7 patients $(2,79 \%)$. Deux patients $(0,80 \%)$ avaient présenté un diverticule œsophagien, et un patient $(0,40 \%)$ une angiodysplasie gastrique. Chez 25 patients (9,96\%), la fibroscopie œsogastroduodénale était normale.

\section{Hémorragies digestives hautes d'origine hypertensive portale}

L'analyse des 69 patients présentant une hémorragie digestive d'origine hypertensive portale avait permis de retrouver comme lésion une 
rupture de varices œsophagiennes chez patients 68 (98,55\%) et une rupture de varices gastriques chez 1 patient. L'âge moyen des patients était de $43,11( \pm 13,96)$ ans [20 ans -80 ans] ; les sujets de sexe masculin représentaient $75 \%$; et la tranche d’âge des [30 ans - 40 ans[ était la plus représentée. L'hypertension portale était d'origine intra-hépatique chez l'ensemble des patients ; et la cirrhose était en cause, suivie de la bilharziose hépatique dans respectivement $96 \%$ et $4 \%$ des cas.

\section{Hémorragies digestives hautes d'origine non hypertensive}

L'analyse des patients présentant une hémorragie digestive haute d'origine non hypertensive portale retrouvait un âge moyen de 46,05 $( \pm 17,64)$ ans [15 ans - 95 ans]. Les hommes représentaient 72,22\% (sex-ratio $\mathrm{H} / \mathrm{F}=2,32$ ). (Tableau 2)

Tableau 2. Étiologies des hémorragies digestives hautes non hypertensives

\begin{tabular}{|c|c|c|c|c|c|}
\hline \multicolumn{2}{|c|}{ Etiologies } & $\begin{array}{c}\text { Age moyen } \\
\text { (années) }\end{array}$ & $\begin{array}{c}\text { Sex-ratio } \\
\text { H/F }\end{array}$ & Effectifs & Pourcentages \\
\hline Esophagite & & $36,88 \pm 18,67$ & 2 & 9 & 5,73 \\
\hline Mallory-Weiss & & $37,90 \pm 20,49$ & 4,5 & 11 & 7 \\
\hline $\begin{array}{c}\text { Diverticule } \\
\text { œsophagien }\end{array}$ & $54,5 \pm 9,5$ & $2 / 0$ & 2 & 1,27 \\
\hline $\begin{array}{c}\text { Gastroduodénites } \\
\text { aiguës }\end{array}$ & & $47,58 \pm 15,41$ & 2,2 & 67 & 42,67 \\
\hline $\begin{array}{c}\text { Angiodysplasie } \\
\text { gastrique }\end{array}$ & 23 & $0 / 1$ & 1 & 0,64 \\
\hline Cancers gastriques & & $56,42 \pm 25,52$ & 6 & 7 & 4,46 \\
\hline $\begin{array}{c}\text { Ulcères } \\
\text { gastroduodénaux }\end{array}$ & $\begin{array}{c}\text { Forrest } \\
\text { I }\end{array}$ & $46,45 \pm 17,04$ & 3,8 & 100 & 63,69 \\
\cline { 2 - 6 } & $\begin{array}{c}\text { Forrest } \\
\text { II }\end{array}$ & & & 2 & - \\
\cline { 2 - 6 } & $\begin{array}{c}\text { Forrest } \\
\text { III }\end{array}$ & & & 8 & - \\
\hline
\end{tabular}

Les lésions siégeaient à l'œsophage, à l'estomac au bulbe et au duodénum dans respectivement $5,88 \%, 41,17 \%, 37,10 \%$ et $0,90 \%$. Les ulcères gastroduodénaux représentaient $54,94 \%$ des hémorragies digestives hautes d'origine non hypertensive portale.

\section{Scores pronostiques}

Le score de Rockall avait été évalué chez les patients à l'admission et après réalisation de la fibroscopie. Le score de Rockall avait une valeur moyenne de $4( \pm 1)$ (extrêmes de 1 et 9 ) chez l'ensemble des patients. La valeur moyenne du score de Rockall était de $4( \pm 1)$ chez les patients présentant une hémorragie d'origine hypertensive et de $3( \pm 1)$ chez les patients avec 
hémorragie non hypertensive portale $(\mathrm{p}<0,001)$. On ne notait pas de différence statistique significative par rapport au sexe. (Tableau 1)

Le score de Glasgow-Blatchford avait été évalué chez les patients à l'admission. On notait une valeur moyenne de $9( \pm 3)$ (extrêmes de 2 et 17) chez l'ensemble des patients. La valeur moyenne du score de Glasgow-Blatchford était de $10( \pm 3)$ chez les patients présentant une hémorragie d'origine hypertensive et $9( \pm 3)$ chez les patients avec hémorragie digestive haute d'origine non hypertensive $(\mathrm{p}<0,001)$. Il n’y avait pas de différence statistique significative par rapport au sexe. (Tableau 1)

\section{Evolution}

L'évolution pendant les 42 jours suivant l'admission avait été marquée par une récidive hémorragique chez 31 patients (12,35\%) et un décès chez 14 patients $(5,58 \%)$. La récidive hémorragique et le décès étaient survenus chez les patients présentant un saignement d'origine hypertensive portale dans respectivement $54,84 \%(p<0,001)$ et $71,42 \%(p<0,001)$. (Tableau 1)

\section{Discussion}

L'hémorragie digestive haute est une urgence médico-chirurgicale dont la prise en charge en Afrique nécessite un plateau technique adéquat. Dans les pays en voie de développement où le plateau technique est défaillant et le personnel insuffisant, il importe de pouvoir identifier les facteurs associés aux différentes étiologies d'une part, et les facteurs de mauvais pronostic d'autres parts, afin de déterminer dès l'admission les patients nécessitant une surveillance particulière.

L'âge moyen était de 45,34( $\pm 16,72)$ ans, avec une prédominance masculine (sex-ratio H/F=2,64). Ces données sont similaires à celles de Gueye et al (Gueye MN et al., 2021) au Sénégal et Okon et al (Okon AJ et al., 2015) en Côte d'Ivoire. Les facteurs de risque de lésions inflammatoires aiguës ou chroniques œsogastroduodénales (prise de médicaments gastrotoxiques, jeûne); et l'évolution lente des hépatopathies chroniques responsables d'hypertension portale expliquent la survenue des hémorragies digestives hautes chez l'adulte jeune. Notons que la moyenne d'âge dans les séries

Africaines était inférieure à celle rapportée par Nahon et al (Nahon S et al., 2014) en France (63 ans) et Cooper et al (Cooper GS et al., 2012) aux USA (66 ans). Cette différence d'âge s'expliquerait par l'existence dans ces pays d'une population vieillissante en rapport avec une espérance de vie plus élevée.

Les patients avaient été classés en deux groupes en fonction des résultats de leurs fibroscopies. Il s'agissait du groupe des HDH d'origine hypertensive portale, et du groupe des HDH d'origine non hypertensive portale. Dans $27,71 \%$ des cas le saignement était d'origine hypertensive 
portale. La majorité des patients présentait une rupture de varices œsophagiennes compliquant une cirrhose. Chez 72,11\% des patients, l'hypertension portale n'était pas responsable du saignement. L'exploration endoscopique avait retrouvé chez ces patients des lésions inflammatoires aiguës ou chroniques des muqueuses de l'appareil digestif haut.

Les résultats de cette étude sont identiques à ceux des séries OuestAfricaines (Katile D et al., 2020; Okon AJ et al., 2015) ; mais diffèrent des données européennes (Cooper GS et al., 2012; Nahon S et al., 2014). L'explication réside dans l'épidémiologie des infections aux virus des hépatites ( $\mathrm{B}$ et $\mathrm{C}$ ) qui sévissent en Afrique sub-saharienne sur un mode endémique, avec contamination en période péri-natale. L’hypertension portale résultant de ces hépatopathies chroniques est ainsi plus fréquente en Afrique sub-saharienne.

La prédominance masculine retrouvée pourrait s'expliquer dans le groupe des HDH hypertensives par une exposition plus élevée des sujets de sexe masculin aux facteurs de risque des hépatopathies (alcoolisme, hépatite virales $B$ et $C$ ). L'anamnèse avait retrouvé comme unique facteur associé à ce groupe l'existence préalable d'une hépatopathie chronique $(\mathrm{p}<0,001)$. La prise d'anti-inflammatoire non stéroïdien était statistiquement associée au groupe des HDH d'origine non hypertensive portale ( $<<0,001)$.

L'éthylisme chronique, le tabagisme et l'existence d'une maladie ulcéreuse œsogastro-duodénale n'étaient pas statistiquement associées au groupe des HDH d'origine non hypertensive portale. Cette différence non significative serait en rapport avec le faible effectif de notre échantillon d'une part, et à l'existence de données manquantes d'autres parts.

Sept patients présentaient un cancer gastrique. L'âge moyen de ces patients était la plus élevée $(56,42 \pm 25,52$ ans), et on notait une forte prédominance masculine. Ces résultats sont superposables à l'épidémiologie des cancers gastriques, qui surviennent chez le sujet âgé, avec une prédominance masculine. Cette prédominance masculine serait en rapport avec une inégale exposition aux facteurs de risque : tabagisme; alcoolisme (Thrumurthy et al., 2013). Le cancer gastrique survenait précocement chez nos patients, comparativement aux séries européennes. La précocité de l'infection à Helicobacter Pylori en Afrique, corrélée à la diminution de cette même infection en Europe serait responsable de cet écart d'âge (Arnold et al., 2013).

L'angiodysplasie est une lésion vasculaire acquise superficielle de la muqueuse. Elles représentent $4 \%$ des étiologies des hémorragies digestives hautes (Moreto et al., 227apr. J.-C.). Dans notre étude, le seul cas d'angiodysplasie représentait $0,64 \%$ des étiologies des hémorragies. Cette faible fréquence est certainement due à l'absence dans notre plateau technique de moyens d'exploration de l'ensemble du grêle. En effet $40 \%$ à $60 \%$ des angiodysplasies digestives sont localisées sur l'intestin grêle (Lecleire et al., 
2012). Les angiodysplasies surviendraient généralement après 60 ans (Boley \& Brandt, 1986), contrairement à notre patient chez qui le diagnostic avait été posé à 29 ans.

La fibroscopie œsogastro-duodénale était normale chez 9,96\% des patients. Plusieurs études similaires (Arka et al., 2019; Hearnshaw et al., 2007; Van Leerdam ME et al., 2003) avaient rapporté que la fibroscopie œsogastroduodénale réalisée dans le cadre de l'exploration d'une hémorragie digestive haute était normale dans 3\% à 17\%. Arka et al (Arka et al., 2019) avaient mené des explorations complémentaires (vidéocapsule endoscopique, entéroscopie double ballon, angioscanner abdominal) qui avaient permis de retrouver la lésion en cause dans $48 \%$ des cas.

Les patients ayant présenté une hémorragie digestive haute d'origine hypertensive portale avaient des scores pronostiques, un taux de récidive hémorragique et de décès plus élevés $(p<0,001)$. La survenue d'une récidive hémorragique et du décès étaient similaires à celles rapportées par Gueye et al (Gueye MN et al., 2021), mais supérieures à celle rapportées par Nahon et al (Nahon S et al., 2014). Ce meilleur pronostic retrouvé dans les séries européennes s'explique par la disponibilité des médicaments adéquats dont l'utilisation est indiquée dans la prise en charge des hémorragies digestives hautes par hypertension portale. L'absence de ces drogues dues à leur coût élevé est responsable d’une morbi-mortalité élevée associée aux hémorragies digestives hautes en Afrique (Gueye MN et al., 2021).

\section{Conclusion}

Les lésions inflammatoires aiguës et chroniques représentent la première étiologie des hémorragies digestives hautes dans le service d'Hépato-gastroentérologie du CHU Campus. Ces hémorragies sont associées à la prise de médicaments gastrotoxiques et à un moindre risque de récidive hémorragique et de décès.

\section{References:}

1. Arka, B., Saptarshi, B., \& Gopal, K. (2019). Acute upper gastrointestinal bleed: An audit of the causes and outcomes from a tertiary care center in eastern India. Indian J Gatroenterol, 38(3), 190-202.

2. Arnold, M., Karim-Kos, H., \& Coebergh, J. (2013). Recent trends in incidence of five common cancers in 26 European countries since 1988: Analysis of the European cancer observatory. Eur J Cancer,51(9),1164-87.

3. Bagny, A., Bouglouga O, \& Djibril, M. (2012). Profil étiologique des hémorragies digestives hautes de l'adulte au CHU-campus de Lomé (Togo). J Afr Hepato Gastroenterol, 6, 38-42. 
4. 4. Boley, S., \& Brandt, L. (1986). Vascular ectasias of the colon. Dig Dis Sci, 31(9), 26-42.

5. Cooper GS, Chak A, \& Way LE. (2012). Early endoscopy in upper gastrointestinal hemorrhage: Associations with recurrent bleeding, surgery, and length of hospital stay. Gastrointest Endosc, 49, 145-152.

6. Czernichow P, Hochain P, Nousbaum JB, Raymond JM, Rudelli A, \& Dupas JL. (2000). Epidemiology and course of acute upper gastrointestinal haemorrhage in four French geographical areas. Eur J Gastroenterol Hepatol, 12, 175-181.

7. D’amigo, G., \& De Franchis, R. (2003). Upper digestive bleeding in cirrhosis. Post-therapeutic outcome and prognostic indocators. Hepatology, 38, 599-612.

8. Gueye MN, Diallo S, Ndiaye BM, Fall MP, Cisse SAB, Diouf G, Bassene ML, Dia D, \& Mbengue M. (2021). Upper gastrointestonal bleeding in Senegal: Preliminary results of a single-centre prospective study. Japanese J of Gastroenterol, 1-4.

9. Hearnshaw, S., Logan, R., \& Lowe, D. (2007). Acute upper gastrointestinal bleeding in the UK: patient characteristics, diagnoses and outcomes in the 2007 UK audit. Gut, 60, 1327-1335.

10. Hervé S. (2007). Epidemiology of upper digestive bleedings: New aspects. Hepato-Gastro, 14, 205-210.

11. Katile D, Sangare S, Traore LI, Sogoba G, \& Cisse SM. (2020). Etiological profile of upper digestive heamorrhages externalized in decentralized environment : Case of the regional hospital of Kayes in Mali. Rev Int Sc Med Abj, 22, 123-126.

12. Lasserre N, Duval F, \& Pateron D. (2009). Upeer digestive bleedings, management into emergency services. Emergencie, 98, 959-968.

13. Lecleire S, Iwanicki-Carron I, \& Di-Fiore A. (2012). Yield and impact of emergency capsule enteroscopy in severe obscure-overt gastrointestinal bleeding. Endoscopy, 44(4), 337-342.

14. Moreto M, Figa M, \& Ojembarrena E. (1986). Vascular malformations of the stomach and duodenum: An endoscopic classification. Endoscopy, 18(6), 227-229.

15. Nahon S, Hagège H, \& Latrive JP. (2014). Epidemiological and prognostic factors involved in upper gastrointestinal bleeding : Results of a French prospective multicenter study. Epidemiological and prognostic factors in UGIB in France. Endoscopy, 44, 214-218.

16. Okon AJ, Thot'o AS, Diakite M, Soro D, Ouattara A, \& Kone A. (2015). Résultats et facteurs prédictifs de mortalité des hémorragies digestives hautes en hospitalisation: Étude multicentrique en Côted'Ivoire. Acta Endosc, 45, 285-290. 
17. Thrumurthy S, Chaudry M, \& Hochhauser D. (2013). The diagnosis and management of gastric cancer. BMJ, 347, 6367.

18. Van Leerdam ME, Vreeberg EM, \& Rauws EAJ. (2003). Acute upper GI bleeding: Did anything change ? Time trend analysis of incidence and outcome of acute upper GI bleeding between 1993/1994 and 2000. Am J Gastroenterol, 98, 1494-1499. 